\title{
Relación entre enfermedad periodontal y parto prematuro. Bajo peso al nacimiento: una revisión de la literatura
}

\author{
FLORES J* \\ OTEO A** \\ MATEOS L** \\ BASCONES A***
}

Flores J, Oteo A, Mateos L, Bascones A. Relación entre enfermedad periodontal y parto prematuro. Bajo peso al nacimiento: una revisión de la literatura. Av Periodon Implantol. 2004; 16, 2: 93-105.

\begin{abstract}
RESUMEN
A pesar de los esfuerzos realizados en el control del embarazo y el parto, la frecuencia de los partos prematuros no ha disminuido en las dos últimas décadas. El parto prematuro supone una seria amenaza para la salud del recién nacido, y representa además un porcentaje muy importante de los costes destinados a la atención sanitaria perinatal. La investigación científica ha revelado diversos mecanismos por los que infecciones remotas como la enfermedad periodontal pueden participar en la patogenia del parto prematuro. Estudios recientes han demostrado que la enfermedad periodontal es un factor de riesgo para el parto prematuro, habiéndose hallado un riesgo relativo mayor que el calculado para factores de riesgo tradicionales como el tabaquismo, bajo peso materno o consumo de alcohol. Se ha sugerido que la prevención y el tratamiento de la enfermedad periodontal en mujeres gestantes podrían contribuir a la prevención del parto prematuro. En el presente artículo revisamos la evidencia científica presente en la literatura acerca de esta asociación y del papel del tratamiento periodontal en la prevención del parto prematuro.
\end{abstract}

\section{PALABRAS CLAVE}

Parto prematuro, bajo peso al nacimiento, enfermedad periodontal, gestación, evidencia científica.

Aceptado para publicación: Marzo 2004

\section{INTRODUCCIÓN}

El parto prematuro, el bajo peso al nacimiento o la restricción del crecimiento fetal durante la gestación son complicaciones del embarazo que pueden conllevar severas consecuencias. Con frecuencia, los niños nacidos bajo estas circunstancias arrastran secuelas de diversa gravedad, pudiendo incluso acontecer la muerte durante los primeros meses de vida.

La Organización Mundial de la Salud define el parto prematuro o pretérmino $(P P)$ como aquel que ocurre antes de cumplirse las 37 semanas de gestación, siendo muy prematuro si se produce antes de las 32 semanas, e inmaduro si es antes de 28 semanas. Por el contrario, gestación a término es aquella que ha cumplido las 37 semanas y será postérmino si dura mas de 42 semanas; la duración media de la gestación es de 40 semanas. Se considera que el recién nacido tiene un bajo peso al nacimiento (BPN) cuando éste no alcanza los 2.500 gramos. Dentro de esta categoría, hablamos de muy bajo peso al nacimiento cuando éste es menor

*** Catedrático de Medicina Bucal y Periodoncia, Facultad de Odontología UCM.
} 
de 1.500 gramos, y será extremadamente bajo si no alcanza los 1000 gramos de peso. Dado que la edad gestacional y el peso al nacimiento se encuentran en relación directa, con frecuencia un recién nacido prematuro tendrá un bajo peso al nacimiento, y nos referiremos entonces a un parto prematuro con bajo peso al nacimiento $(P P / B P N)$ (World Health Organization 1950).

Aunque en las últimas décadas la supervivencia de los recién nacidos prematuros ha aumentado espectacularmente, incluso en los de peso y edad gestacional más bajos, aproximadamente un $20 \%$ de estos niños sufre secuelas importantes, tales como parálisis cerebral, leucomalacia periventricular (precursor histológico de la parálisis cerebral), displasia broncopulmonar, distrés respiratorio, retinopatía de la prematuridad, déficit visual grave o pérdida de la audición (Hack y cols. 1994, Hack y cols 1995, Paquette y cols. 1999). Assimismo, estos niños sufren más infecciones y de mayor gravedad, siendo frecuente el reingreso por infecciones respiratorias bajas (especialmente bronquiolitis) durante el primer año. De hecho, el parto pretérmino constituye la causa principal de morbimortalidad perinatal, así como de discapacidad neurológica a largo plazo (Lorenz y cols. 1998). Actualmente los recién nacidos prematuros representan el $7-8 \%$ de los nacidos vivos, suponen más del $60 \%$ de los casos de mortalidad neonatal y a ellos se dedica más del $50 \%$ de los costes destinados a la atención sanitaria perinatal. Los nacimientos de bajo peso suman el 10\% del total, y los de muy bajo peso el $1.2 \%$. La incidencia de PP/BPN no ha disminuido en las dos últimas décadas, y la causa subyacente es habitualmente desconocida (Jeffcoat y cols 2001, Krueger y Scholl 2000, Stevenson y cols. 1998, Paquette y cols 1999).

En el Hospital Clínico de San Carlos (Madrid) se registraron entre 1988 y 2001 un total de 25.819 partos, de los cuales casi 2.500 (el 9.6\%) fueron pretérminos. De estos recién nacidos prematuros, 803 $(32.12 \%)$ pesaron más de 2.500 gramos; 1.133 $(45.32 \%)$ tuvieron un peso de entre 1.500 y 2.500 gramos; 337 (13.48\%) entre 1.000 y 1.500 gramos, y 223 $(8.92 \%)$ no alcanzaron los 1.000 gramos (1).

Algunos autores opinan que existe poca evidencia acerca de las causas del PP/BPN, a pesar de los numerosos estudios y revisiones destinados a investigar esta cuestión (Alexander y Korenbrot 1995, Offenbacher y cols. 1998). Se conocen diversos factores de riesgo asociados al parto prematuro (tabla 1).

La colonización vaginal por especies del género Bacteroides aumenta la tasa de PP/BPN un 40-60\% (Minkoff y cols. 1984, McDonald y cols.1991), mientras que haber sufrido un PP/BPN previamente multiplica por tres el riesgo de sufrirlo de nuevo (Offenbacher y cols. 2001). Se ha detectado también una cierta tendencia familiar, lo que hace sospechar que pueda existir un componente genético asociado a la patogénesis del parto prematuro/bajo peso al nacimiento (Offenbacher y cols. 2001).

\section{FACTORES DE RIESGO ASOCIADOS A PP/BPN}

- Consumo de tabaco, alcohol y/o drogas durante el embarazo.

- Enfermedades asociadas a la gestación: infecciones del tracto genitourinario, hipertensión, anemia, diabetes gestacional.

Cuidados prenatales inadecuados.

- Edad de la madre menor de 17 o mayor de 34 años.

- Raza afroamericana.

- Embarazo múltiple.

Infecciones fetales.

Primer embarazo.

- Trabajo con esfuerzo físico importante o con un elevado nivel de estrés.

Bajo peso materno.

Baja estatura materna.

PP/BPN previo.

Cuello uterino corto.

Estatus socioeconómico bajo.

Nutrición materna pobre.

- Diversos desórdenes metabólicos y genéticos.

- Madre nacida prematuramente.

Tabla 1. Factores de riesgo conocidos asociados al parto prematuro/bajo peso al nacimiento. (Paquette y cols. 1999, Offenbacher y cols. 1998 , Offenbacher y cols. 2001). Datos facilitados por el Dr. Pluvio Coronado, del Servicio de Obstetricia y Ginecología del Hospital Clínico de San Carlos, Madrid. 
Las infecciones subclínicas han sido señaladas como una causa importante del parto prematuro, especialmente en los muy prematuros. Gibbs enumera las evidencias que avalan esta afirmación (Gibbs 2001):

1. La prevalencia de corioamnionitis histológica aumenta entre los casos de parto prematuro.

2. Se han observado evidencias de infección clínica en madre y recién nacido tras el parto prematuro.

3. Se ha hallado una asociación significativa entre la infección del tracto genital bajo (o sus patógenos) y el parto prematuro o ruptura prematura de las membranas placentarias.

4. Se han obtenido cultivos positivos de líquido amniótico o membranas placentarias de pacientes que han tenido un parto prematuro.

5. Se han detectado marcadores de infección en casos de parto prematuro.

6. Las bacterias o sus productos inducen el parto prematuro en modelos animales.

7. Ensayos con antibióticos mostraron una menor tasa de parto prematuro o difirieron el parto.

No obstante otros estudios no han mostrado ningún efecto preventivo por parte de la terapia antibiótica en la disminución de estas complicaciones del embarazo (Morales y cols. 1994). Se ha sugerido que estos confusos resultados pueden deberse a diferencias entre las poblaciones estudiadas respecto a la sus- ceptibilidad al parto prematuro o a un control ineficaz de las infecciones (Stetzer y cols. 2000).

Se han relacionado con el parto prematuro infecciones como las del tracto genitourinario, la pielonefritis aguda, la pneumonía (en la era preantibiótica) o la enfermedad periodontal (EP) (Gibbs 2001, Jeffcoat y cols. 2001 a). La existencia de una asociación entre esta última y el parto prematuro ha sido ampliamente estudiada y documentada en los últimos años, convirtiéndose en un importante tema de investigación en el campo de la medicina periodontal. Esta cuestión es además de gran trascendencia para cualquier odontólogo o estomatólogo, puesto que debe existir un criterio común en cuanto a si se debe tratar a las pacientes embarazadas con enfermedad periodontal, y, en caso afirmativo, qué tratamiento es el más adecuado.

El objetivo del presente artículo es revisar la literatura disponible acerca de la relación entre enfermedad periodontal y parto prematuro/bajo peso al nacimiento, analizando la evidencia científica existente respecto a los factores que relacionan ambas entidades y la importancia de aplicar o no un tratamiento a pacientes susceptibles de sufrir un parto prematuro.

\section{MECANISIMOS PATOGÉNICOS}

Los mecanismos por los que las infecciones en la madre pueden provocar el parto prematuro o participar en la cadena de eventos que conducen a él han sido ampliamente estudiados, y pueden resumirse en el esquema de la figura 1.

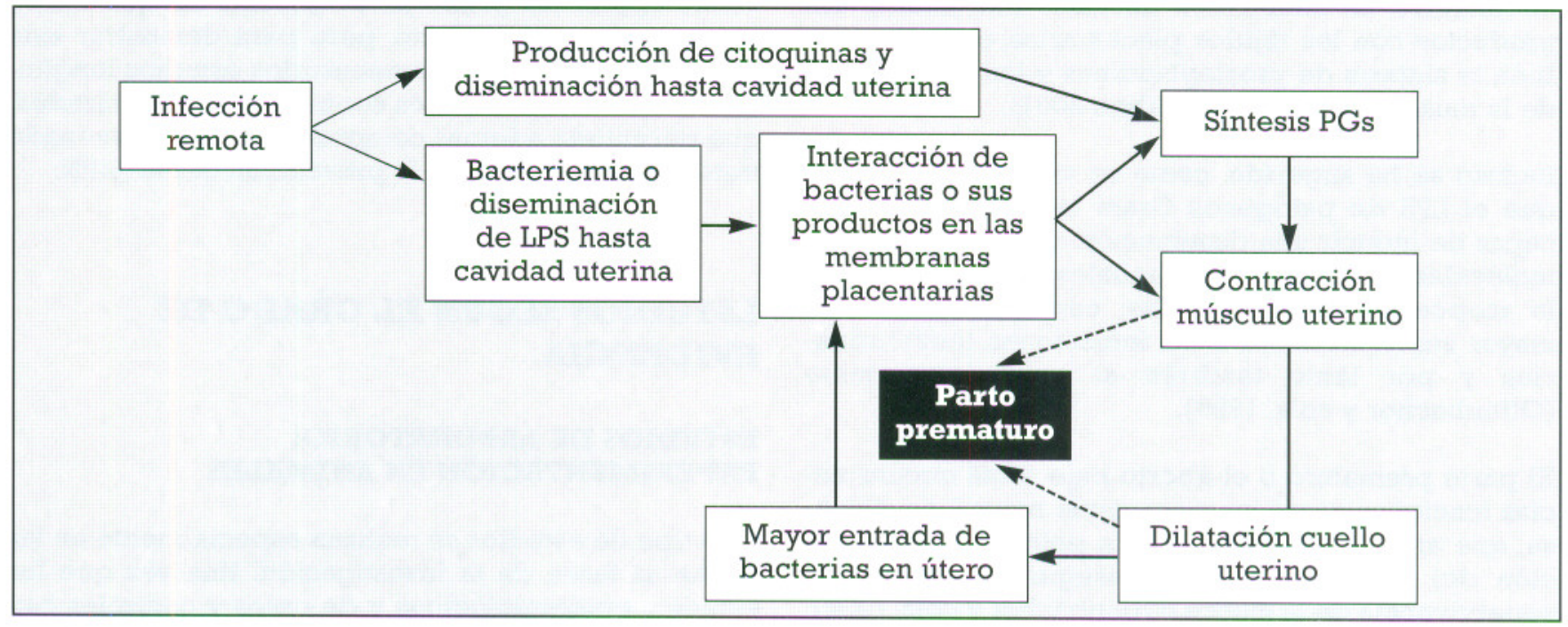

Figura 1. Mecanismos mediante los que una infección remota puede afectar al embarazo y desencadenar el parto prematuro. 
En una primera vía, las citoquinas y otros mediadores inflamatorios generados en la respuesta inmune frente a la infección se diseminarían por vía sanguínea hasta alcanzar la cavidad uterina, donde promoverán la síntesis de prostaglandinas (PGs). Entre los diversos efectos producidos por estos mediadores de la inflamación se encuentran los siguientes: la prostaglandina E2 (PGE2) provoca estrés oxidativo, contracción del músculo liso y oxidación de las lipoproteínas de baja densidad (LDL), mientras que la interleuquina $1 \beta$ (IL-1 $\beta$ ), el factor de necrosis tumoral a (TNF $\alpha$ ) y la interleuquina 6 (IL-6) son capaces de estimular la adhesión endotelial, hiperlipidemia, liberación hepática de reactivos de fase aguda y catabolismo del tejido conectivo entre otros efectos. Muchos de estos eventos están implicados en la historia natural del parto prematuro (Paquette y cols. 1999). Algunos estudios han mostrado que la cantidad de IL-6 en líquido amniótico se ve aumentada cuando el parto se produce antes de las 34 semanas de gestación (Hillier y cols. 1993), y que la concentración de IL-6 en líquido amniótico es un marcador fiable de infección en mujeres que sufren un parto prematuro o ruptura prematura de membranas (Romero y cols. 1993). Se ha sugerido también que la IL-6 estimula la producción de prostaglandinas por las propias membranas placentarias (Jeffcoat y cols. 200la). Las contracciones de la musculatura uterina provocadas por las prostaglandinas conducen a una dilatación del cuello uterino que directamente podría desencadenar el parto, y que en cualquier caso facilita la entrada de más bacterias al útero, cerrando un círculo vicioso que finalmente concluye con el parto prematuro.

Por otra parte, la infección podría generar una bacteriemia transitoria o bien productos bacterianos como el lipopolisacárido (LPS) podrían alcanzar el torrente circulatorio. La interacción de estas bacterias o sus productos con los tejidos placentarios estimula también la síntesis de prostaglandinas y las contracturas de la musculatura uterina (Gibbs 2001).

Incluso se ha sugerido, como se verá más adelante, que el LPS de patógenos Gram negativos orales es capaz de inducir una disminución en la expresión de moléculas de adhesión endoteliales que participan en la respuesta inmune celular, confiriendo así una mayor susceptibilidad a las infecciones genitourinarias y por tanto también al parto pretérmino (Offenbacher y cols. 1996).

El parto prematuro o el aborto bajo estas circunstancias funcionan como un mecanismo natural de defensa, que ante la amenaza de una peligrosa complicación del embarazo trata de asegurar al menos la supervivencia de la madre (Offenbacher y cols. 1998). La enfermedad periodontal es una patología infecciosa causada predominantemente por bacterias Gram negativas, y puede por tanto funcionar como factor de riesgo para el parto prematuro a través de los citados mecanismos (Gibbs 2001, Offenbacher y cols. 1998, López y cols. 2001). Se ha demostrado que maniobras como el cepillado dental o el raspado y alisado radicular son capaces de producir una bacteriemia transitoria (Lofthus y cols. 1991, Waki y cols. 1990), lo que ocurrirá con más frecuencia cuanto mayor sea el grado de inflamación de los tejidos periodontales, y existen por tanto mecanismos biológicamente plausibles para esta asociación (Jeffcoat y cols. 2001 a, Offenbacher y cols. 1998). Una vez formulada la hipótesis y demostrada la plausibilidad biológica, serán los estudios acerca de este tema los que aportarán la evidencia necesaria para demostrar o desmentir la existencia de esta asociación. Pero ¿qué estudios son más adecuados para analizar esta cuestión? ¿Cuáles aportan más evidencia? En primer lugar debemos saber que el estudio de los partos prematuros plantea importantes dificultades de carácter ético. Por otro lado no es fácil conseguir una muestra relativamente grande, y hay además un elevado número de factores que intervienen en las complicaciones del embarazo y que actúan como factores de confusión (Jimeno y cols. 1997). Todo esto hace que sea compleja tanto la realización de estos estudios como la interpretación de los resultados. En segundo lugar debemos tener en cuenta que, como es lógico, no todos los estudios tienen el mismo valor. Existe una jerarquía entre los distintos tipos de estudios en base a la evidencia científica que aportan y por tanto a su valor científico (Woolf y cols. 1990, Sackett 1993). Como se verá mas adelante, los estudios epidemiológicos y de casos y controles permiten establecer una asociación entre la enfermedad periodontal y el PP/BPN. Una vez hallados los mecanismos biológicamente plausibles que explican esta asociación, la experimentación en animales aporta las primeras evidencias de que existe una relación causa-efecto, pero para demostrar esta relación en humanos son necesarios estudios longitudinales y estudios clínicos controlados randomizados, que permitirán además comprobar si un determinado tratamiento es eficaz en la prevención del PP/BPN.

\section{ESTUDIOS SEGÚN EL GRADO DE EVIDENCIA}

\section{ESTUDIOS DE LABORATORIO: EXPERIMENTACIÓN EN ANIMALES}

Este tipo de estudios se realizan especialmente en las primeras fases de la investigación. Una vez que los estudios epidemiológicos y de casos y controles han indicado la existencia de una asociación, en la experimentación animal se obtienen a menudo las prime- 
ras evidencias de la presencia de una relación causaefecto. Los hallazgos de estos estudios no son completamente extrapolables a la fisiología o patología humanas, por lo que el grado de evidencia que se les atribuye es relativamente bajo.

Partiendo de que las infecciones del tracto genitourinario pueden provocar indirectamente el PP/BPN, Collins y colaboradores se plantearon si una infección periodontal puede causarlo también por los mismos mecanismos indirectos. Para explorar esta hipótesis escogieron un modelo de hamsters embarazadas a las que inocularon subcutáneamente un patógeno periodontal en una cámara de acero inoxidable, generando así una infección subclínica, crónica y localizada similar a la periodontitis. La muestra fue dividida en cuatro grupos: en un primer grupo se inoculó Porphyromonas gingivalis muerta por calor (a modo de inmunización previa a este patógeno) y tres semanas después se inoculó $P$. gingivalis viva; un segundo grupo recibió solamente $P$. gingivalis viva, mientras que al tercero se le administró sólo $P$. gingivalis muerta y un cuarto grupo sirvió de control, recibiendo únicamente el vehículo. Una vez ocurrido el parto en todos los especímenes se observó que los animales a los que se había inoculado $P$. gingivalis viva y muerta o bien sólo $P$ gingivalis viva tuvieron crías de un peso entre un 15 y un $18 \%$ menor. La inmunización previa mediante administración del organismo muerto no logró por tanto conferir una protección a la hora de prevenir el bajo peso al nacimiento. Se observaron también cantidades elevadas de $\mathrm{PGE}_{2}$ y TNF- $\alpha$ en el fluido aspirado de las cámaras subcutáneas (Collins y cols. 1994).

Dando continuidad a estos esperanzadores hallazgos, Collins y cols. realizaron en 1995 un estudio en hembras de hamster embarazadas en las que se indujo experimentalmente una infección periodontal. Los animales fueron divididos en cuatro grupos, dos de ellos se alimentaron con una dieta control y los otros dos con una dieta que promoviera la formación de placa bacteriana. A un grupo de cada tipo se le administró una cantidad de $P$. gingivalis exógena mediante sonda gástrica. No se hallaron diferencias estadísticamente significativas entre cada subgrupo con $P$. gingivalis y su subgrupo corespondiente de dieta similar. Sin embargo, los dos subgrupos alimentados con la dieta estimulante de la formación de placa, con o sin $P$. gingivalis, desarrollaron periodontitis, hecho que se confirmó histológicamente. Las crías que nacieron de las hembras de hamster pertenecientes a estos dos subgrupos tuvieron un peso al nacimiento significativamente menor (un $22.5 \%$ menor en comparación con los subgrupos sin periodontitis, $\mathrm{p}=0.037$ ) y mostraron un aumento significativo en la concentración de $\mathrm{PGE}_{2}$ y TNF- $\alpha$ en líquido amniótico, mediadores inflamatorios capaces de inducir contracciones uterinas, dilatación del cuello uterino, parto prematuro y aborto (Collins y cols. 1995).

\section{ESTUDIOS DE CASOS Y CONTROLES}

Los primeros estudios realizados en el proceso de la investigación científica tras la formulación de una hipótesis son a menudo los de casos y controles. Estos estudios son relativamente rápidos, económicos y facilitan la tarea de conseguir sujetos puesto que no exigen un número elevado de ellos y sólo se requiere una única exploración por individuo, por lo que no es necesario retenerlos; (Muñiz y Gabriel 2003, Jeffcoat 2001 a). Además los estudios de casos y controles son los únicos posibles en ciertas situaciones, en particular en la investigación de enfermedades raras (Muñiz y Gabriel 2003). El grado de evidencia científica que aportan es superior que el de los estudios en animales. Por otro lado presentan el inconveniente de que su diseño retrospectivo no permite establecer una temporalidad en la sucesión de los eventos, al contrario de lo que sucede con los estudios prospectivos (Jeffcoat 2001 a).

En un estudio de casos y controles en 55 parejas de mujeres, Dasanayake observó que las madres con salud periodontal tenían un menor riesgo de dar a luz un recién nacido de bajo peso, con un riesgo relativo de 0.3 (Dasanayake 1998).

Offenbacher y su grupo de la Universidad de North Carolina llevaron a cabo en 1996 el primer estudio que logra evidenciar en humanos la asociación entre periodontitis y parto prematuro/bajo peso al nacimiento. Se trata de un estudio de casos y controles en 124 mujeres embarazadas o postparto; Los casos de PP/BPN mostraron una pérdida de inserción significativamente mayor que los controles (parto a término/peso normal al nacimiento). Se halló una fuerte significación estadística, situando a la enfermedad periodontal como factor de riesgo para PP/BPN con un riesgo relativo ( $R R$ ) de 7.9 (7.5 para los casos de $\mathrm{PP} / \mathrm{BPN}$ en madres primíparas), habiendo ajustado los demás factores de riesgo conocidos. Este RR fue incluso mayor que el hallado para otros factores de riesgo como consumo de alcohol, tabaquismo o edad de la madre. Los datos del estudio sugieren además que un $18.2 \%$ de los casos de PP/BPN pueden ser atribuíbles a un deficiente estado periodontal materno. Los autores revisaron además los posibles mecanismos por los que se puede producir la interacción entre periodontitis y embarazo. La infección periodontal puede funcionar como reservorio de lipopolisacárido (LPS), que podría alcanzar las membranas placentarias a través del torrente sanguíneo. Se ha demostrado que el LPS estimula la producción de IL-1 $\beta$ y $\mathrm{PGE}_{2}$ mediante células coriamnióticas y trofoblásticas, y 
este hecho a su vez se asocia a menudo al parto prematuro. Por otro lado, los propios mediadores inflamatorios producidos en el periodonto inflamado como $\mathrm{PGE}_{2} \circ \mathrm{TNF}-\beta$ pueden alcanzar el torrente sanguíneo y llegar así a la placenta. Se considera también la posibilidad de que existan factores de confusión genéticos o ambientales: una condición subyacente que predisponga a la madre a sufrir enfermedad periodontal y PP/BPN. Este podría ser el caso de una respuesta inflamatoria hipersensible, que aumentaría el riesgo de sufrir PP/BPN y también una periodontitis más agresiva. Otro posible mecanismo consistiría en la inhibición del aclaramiento normal de microorganismos entéricos por neutrófilos debido a una disminución en la expresión del receptor endotelial Eselectina por parte de las células endoteliales. Se ha demostrado que el LPS producido por patógenos orales es capaz de inducir dicha reducción en la expresión de E-selectina. Al no producirse un infiltrado normal de neutrófilos, microorganismos Gram-negativos podrían invadir el tracto genitourinario y originar una infección que afecte adversamente al embarazo. De este modo, el LPS procedente de patógenos periodontales y liberado al torrente sanguíneo puede aumentar la susceptibilidad a una infección genitourinaria (Offenbacher y cols. 1996).

El grupo de North Carolina compara en un nuevo estudio transversal de casos y controles de 1998 los niveles de inflamación gingival y el estado periodontal de madres prematuras y no prematuras, tratando una vez más de encontrar una relación entre la patología periodontal y los mecanismos que conducen al parto prematuro. En este caso se trató de evaluar los niveles de $\mathrm{PGE}_{2}$ e IL-1 $\beta$ en el fluido gingival de 44 madres en un plazo de tres días después del parto. Se tomaron también muestras de placa bacteriana para realizar sobre ellas técnicas de hibridación mediante sondas de $A D N$, con la intención de detectar y cuantificar la presencia de cuatro patógenos periodontales: Actinobacillus actinomycetemcomitans, $P$ gingivalis, Bacteroides forsythus y Treponema denticola. Además se realizó un exámen periodontal en cada paciente, midiéndose la profundidad de sondaje, los niveles clínicos de inserción y el sangrado al sondaje. Los resultados mostraron una ligera diferencia entre el estado periodontal de los dos grupos, que no fue estadísticamente significativa $(\mathrm{p}=0.1)$ posiblemente, tal y como indican los autores, debido al pequeño tamaño de la muestra. Los niveles de $\mathrm{PGE}_{2}$ en fluido gingival sí fueron significativamente mayores $(p=0.02)$ en los casos de PP/BPN (131.4 ng/ml) frente al grupo control con parto a término y peso normal al nacimiento (62.6 $\mathrm{ng} / \mathrm{ml}$ ). Se observó además en las madres primíparas una relación inversamente proporcional entre los niveles de $\mathrm{PGE}_{2}$ en fluido gingival materno y el peso del recién nacido $(p=0.029)$, y la misma relación inversa se halló también respecto a la edad gestacional del niño en el momento del nacimiento. En el caso de la IL$1 \beta$, si bien los niveles fueron más elevados en las madres prematuras, no hubo diferencias estadísticamente significativas entre ambos grupos $(p=0.21)$, cuestión en la que los autores apelan de nuevo al tamaño muestral. En cuanto a las pruebas microbiológicas, entre las madres que sufrieron $\mathrm{PP} / \mathrm{BPN}$ se hallaron cantidades significativamente mayores para los cuatro patógenos testados, siendo $B$. forsythus el que mostró las diferencias más significativas alcanzando una $\mathrm{p}=$ 0.0001 . Los autores señalan que los niveles elevados de los mediadores inflamatorios en el fluido gingival materno indican no sólo la presencia de enfermedad periodontal, sino que esta además se encuentra en una fase activa, lo que incrementa la posibilidad de exposición sistémica al LPS de los Gram-negativos orales y a los propios mediadores inflamatorios durante el embarazo, resultando en un mayor riesgo de sufrir PP/BPN (Offenbacher y cols. 1998).

En otro estudio de casos y controles de 1999 desarrollado por el grupo de Offenbacher se investigó el aspecto microbiológico de esta asociación. Para ello analizaron muestras de sangre de cordón umbilical de 21 recién nacidos prematuros/de bajo peso y de 39 recién nacidos con peso normal y que completaron la gestación normal. Mediante checkerboard inmunoblotting investigaron la presencia de anticuerpos IgM específicos frente a 13 patógenos periodontales. El $17.9 \%$ de las muestras de niños no prematuros fueron positivas para dichos anticuerpos, mientras que entre los casos de PP/BPN lo fueron un 33.3\%. Asimismo se detectó un predominio de IgM frente a Campylobacter rectus seguido de $P$. gingivalis y Fusobacterium nucleatum. Por consiguiente, estos datos evidencian que la infección periodontal en la madre puede desencadenar una respuesta inmune específica por parte del feto y suponer por tanto una amenaza para él (Offenbacher y cols. 1999).

\section{ESTUDIOS DE COHORTES}

Entre las características de los estudios de cohortes se encuentran la direccionalidad anterógrada (estudios prospectivos), una temporalidad concurrente con la de la historia natural de la enfermedad y una menor posibilidad de sesgos (como los de selección), que por otro lado pueden afectar seriamente a los de casos y controles. Los estudios prospectivos sí permiten establecer una temporalidad en la sucesión de los eventos y sugerir así una relación causa-efecto. Por estos motivos la información proveniente de los estudios de cohortes se considera más fiable que la proporcionada por los de casos y controles. Como inconvenientes cabe decir que son caros, largos y exigen la retención de los sujetos estudiados (Muñiz y Gabriel, 2003). 
A finales del 2001 se publicó un nuevo trabajo del grupo de Offenbacher, un importante y extenso estudio prospectivo a cinco años denominado OCAP (Oral Conditions And Pregnancy). Sobre una muestra de 812 embarazadas se investigó la relación entre el estado periodontal de la madre y la edad gestacional al nacimiento y peso al nacimiento del niño, una vez controlados los factores de riesgo y de confusión habituales, destacándose la importancia de la progresión de la enfermedad periodontal durante el embarazo. Para ello se realizó una clasificación según el estado periodontal de las madres en tres niveles: salud periodontal, enfermedad periodontal leve y enfermedad periodontal moderada-severa, y se llevaron a cabo exámenes periodontales preparto y postparto. Los resultados proporcionaron una gran cantidad de datos, que son contrastados además con una serie de características de la madre: raza, edad, estado civil, tabaquismo, dieta, parto prematuro previo, primer embarazo, vaginosis bacteriana, corioamnionitis, estado periodontal y progresión de la enfermedad periodontal durante el embarazo. En este sentido cabe destacar que casi un tercio $(32.4 \%)$ de las madres de raza negra sufrieron parto prematuro, que un $29.8 \%$ de las madres que sufrieron esta vez un parto prematuro ya lo habían experimentado en embarazos previos, que la prevalencia de EP moderada-severa fue significativamente mayor entre los casos de PP/BPN y que en un $47,2 \%$ de las madres prematuras se detectó progresión de la enfermedad periodontal durante el embarazo. Se halló significación estadística para estas asociaciones, siendo especialmente fuerte para la progresión de EP durante el embarazo ( $p=0.0012)$, entendiendo como tal un aumento de $2 \mathrm{~mm}$ o más en la profundidad de sondaje en 4 o más sitios. Llama también la atención el hecho de que un $28.8 \%$ de todas las madres, prematuras o no, experimentaron un empeoramiento de su estado periodontal durante el embarazo.

La prevalencia de EP materna previa al parto fue significativamente mayor en los casos de gestación menor o igual a 35 semanas. Asimismo, la tasa de PP/BPN fue mucho más elevada entre las madres con EP moderada-severa, de las que un $40 \%$ dieron a luz antes de las 37 semanas de gestación. Hubo 6 casos de muerte neonatal, todos ellos fueron partos prematuros y 5 de ellos en madres con EP moderada-severa. Por otra parte, los investigadores detectaron una tendencia familiar, lo que siembra la sospecha de que exista un componente genético en la patogénesis del PP/BPN. Asimismo, se sugiere que exposiciones intermitentes a corto plazo a factores de riesgo durante el embarazo pueden jugar un papel importante en esta cuestión.

Una segunda parte del estudio contempla el punto de vista microbiológico e inmunológico a través de una evaluación de la respuesta de anticuerpos frente a patógenos orales tanto en la madre como en el feto. La detección de IgM específica en muestras de sangre del cordón umbilical sugiere una exposición directa del feto en el útero a agentes infecciosos, puesto que la IgM materna, al contrario de lo que ocurre con la IgG, no atraviesa la barrera placentaria, deduciéndose que las IgM detectadas fueron producidas por el propio feto. La elevada cantidad de IgM específica frente a Campylobacter rectus detectada en las muestras apunta a una mayor capacidad de este organismo para alcanzar el feto. Es sabido que algunas especies no humanas del género Campylobacter son capaces de atravesar la barrera placentaria e inducir el parto prematuro y/o el aborto en animales de ganado. Otro hallazgo importante es el hecho de que las madres que no desarrollaron una respuesta humoral sistémica frente a patógenos periodontales, especialmente frente a los del Grupo Rojo (P. gingivalis, B. forsythus y T. denticola) (Socransky y cols. 1998), tuvieron una tasa significativamente mayor de partos prematuros, lo que sugiere que dicha respuesta de anticuerpos puede tener una función protectora para el feto. Esto a su vez plantea la posibilidad de que un tratamiento que refuerce la respuesta de anticuerpos por parte de la madre, como el raspado o alisado radicular o incluso la vacunación, podría reducir los efectos adversos de la enfermedad periodontal sobre el embarazo.

Así pues, se concluye que este estudio aporta evidencia de que la EP materna y/o su progresión durante el embarazo constituyen un factor de riesgo para PP/BPN, que esta relación es dosis-dependiente y que la asociación es tanto más fuerte cuanto menor es la edad gestacional al nacimiento, jugando un papel importante la respuesta inmune humoral desarrollada por la madre. Desde esta perspectiva se plantea el diagnóstico y tratamiento periodontal como una importante estrategia preventiva de cara al parto prematuro/bajo peso al nacimiento (Offenbacher y cols. 2001, Madianos y cols. 2001).

Jeffcoat y colaboradores realizaron otro estudio prospectivo en 1313 mujeres embarazadas. Los resultados mostraron un mayor riesgo de PP en pacientes con enfermedad periodontal, con un riesgo relativo de 2.83 para aquellas con EP leve o moderada y de 4.18 para los casos de EP severa respecto al grupo con un estado periodontal de salud. Se calcularon riesgos relativos para las pacientes con EP generalizada y PP a distintas edades gestacionales, obteniéndose los siguientes valores: $R R=4.45$ para $P P$ por debajo de las 37 semanas de gestación, $R R=5.28$ para $P P$ con menos de 35 semanas y $R R=7.07$ para $P P$ que no alcanza las 32 semanas de gestación (Jeffcoat y cols. 2001 a, Jeffcoat y cols. 2001 b). 


\section{ESTUDIOS DE INTERVENCIÓN}

Dentro de los estudios de intervención existen distintos diseños. De ellos, el más alto grado de evidencia se atribuye a los ensayos clínicos controlados randomizados a doble ciego. Estos estudios pueden ofrecer una información de gran calidad en lo referente al efecto de un factor sobre una enfermedad. Esta información es a menudo la que permite modificar los protocolos de tratamiento de una patología. Sin embargo, en ocasiones las propias características de la enfermedad o del factor en cuestión no permiten cumplir sus requisitos (por ejemplo puede ser imposible mantener el doble ciego o la aleatorización), y en otras es la naturaleza de la pregunta investigada la que hace inadmisible este diseño. Por ejemplo, no podemos investigar si la administración de dietilestilbestrol es un factor de riesgo para el parto prematuro puesto que se sabe que produce cáncer ginecológico en las hijas de pacientes tratadas en el pasado (Muñiz y Gabriel, 2003).

Mitchell-Lewis y cols. examinaron en un estudio controlado no randomizado la relación entre las infecciones periodontales y el parto pretérmino, y el efecto del tratamiento periodontal sobre esta complicación del embarazo en un grupo de mujeres jóvenes embarazadas o posparto. Evaluaron la presencia de placa bacteriana, cálculo, sangrado al sondaje y la profundidad de sondaje. Se tomaron muestras de placa subgingival en 145 sujetos (4 muestras por sujeto) y fueron analizadas mediante técnicas de hibridación de sondas de ADN para 12 especies bacterianas. De 164 mujeres que dieron a luz, un grupo de 74 fueron sometidas a profilaxis oral durante el embarazo y las 90 restantes no recibieron tratamiento periodontal prenatal. Hubo un total de 27 casos de PP/BPN (16.5\%). No se observaron diferencias significativas entre el estado periodontal de las madres prematuras y de las no prematuras. Sin embargo, entre los casos de PP/BPN sí hubo niveles significativamente superiores de $B$. forsytus y C. rectus, así como recuentos bastante elevados de otras especies. El PP/BPN ocurrió en un $18.9 \%$ de las mujeres que no recibieron tratamiento periodontal (17 casos) y en un $13.5 \%$ (10 casos) de las que sí lo recibieron. La diferencia no fue estadísticamente significativa, pero en el grupo tratado se observó una reducción del $28.6 \%$ en la incidencia de $\mathrm{PP} / \mathrm{BPN}$ respecto al grupo no tratado (Mitchell-Lewis y cols. 2001).

Otro estudio de intervención fue llevado a cabo por López y colaboradores en 2001 . Se trata de un estudio controlado randomizado sobre una muestra de cuatrocientas mujeres con enfermedad periodontal moderada o severa. Las pacientes fueron asignadas aleatoriamente mediante moneda al aire a un grupo experimental, al que se realizó tratamiento periodontal antes de las 28 semanas de gestación, o a un grupo control, al que se aplicó la terapia periodontal después del parto, obteniéndose 200 pacientes en cada grupo sin que hubiera diferencias significativas entre el estatus periodontal de ambos grupos. Todas las pacientes estudiadas pertenecían a un estatus socioeconómico bajo y estaban integradas en un programa gratuito de cuidados prenatales. Tras completarse un mínimo de 160 partos en cada grupo, se hizo un análisis preliminar de los datos con el objetivo de interrumpir el reclutamiento de pacientes por razones éticas si se hallaba significación estadística en las diferencias entre ambos grupos respecto a la tasa de PP/BPN, hecho que efectivamente ocurrió. En ese momento había 400 pacientes incluídas en el estudio, de las cuales 49 (37 en el grupo experimental y 12 en el grupo control) fueron excluidas del análisis estadístico por distintos motivos. Por tanto se estudiaron 163 partos en el grupo experimental y 188 en el grupo control. A todas las pacientes se les realizó un examen periodontal completo, determinándose las siguientes variables: nivel de higiene oral (en base al porcentaje de localizaciones con acúmulo de placa), inflamación gingival, profundidad de sondaje y nivel de inserción clínica. Las mediciones clínicas fueron llevadas a cabo por dos periodoncistas previamente calibrados hasta lograr un nivel de concordancia adecuado. El tratamiento periodontal consistió en raspado y alisado radicular e instrucciones de control de placa, tras lo que se realizaron sesiones de mantenimiento cada 2 ó 3 semanas hasta ocurrir el parto. Además, en 29 pacientes del grupo experimental que mostraron una periodontitis agresiva severa se aplicó terapia antibiótica (metronidazol $250 \mathrm{mg}$ y amoxicilina $500 \mathrm{mg} 3$ veces al día durante una semana). Al final del tratamiento se realizó un nuevo examen periodontal.

Hubo 22 casos de PP/BPN entre los 351 recién nacidos vivos $(6.26 \%)$. En el grupo experimental se produjeron 3 partos prematuros/de bajo peso de 163 estudiados $(1.84 \%)$, mientras que en el grupo control fueron 19 de 188 (10.11\%), hallándose un riesgo relativo de 5.49 y una $p=0.001$. Los resultados de distintos análisis estadísticos mostraron que las mujeres con enfermedad periodontal tuvieron una tasa de incidencia de PP y de PP/BPN más de 5 veces superior a la de las mujeres periodontalmente sanas. La tasa de bajo peso al nacimiento también fue más alta en las madres con enfermedad periodontal, aunque la diferencia no fue estadísticamente significativa. Se calcularon los siguientes riesgos relativos: para EP y PP, OR $=6.10$; para $\mathrm{EP}$ y $\mathrm{BPN}, \mathrm{OR}=6.96$; para $\mathrm{EP}$ y $\mathrm{PP} / \mathrm{BPN}, \mathrm{OR}=6.67$. Las mujeres que sufrieron el parto prematuro mostraron un estado periodontal significativamente peor y una inflamación gingival significativamente más severa y extendida que las mujeres que tuvieron un parto a término. Se realizó una comparación entre los riesgos relativos de los distintos factores de riesgo cono- 


\begin{tabular}{|c|c|c|c|c|}
\hline & $\begin{array}{c}\text { Prof. + placebo } \\
(n=123)\end{array}$ & $\begin{array}{c}\text { RAR + placebo } \\
(\mathbf{n}=123)\end{array}$ & $\begin{array}{c}\mathbf{R A R}+\text { metronidazol } \\
(\mathrm{n}=120)\end{array}$ & $\begin{array}{l}\text { Grupo ref. } \\
(n=723)\end{array}$ \\
\hline $\mathrm{PP}<37$ semanas & $8.9 \%$ & $4.1 \%(p=0.12) *$ & $12.5 \%(\mathrm{p}=0.37)^{*}$ & $12.7 \%$ \\
\hline $\mathrm{PP}<35$ semanas & $4.9 \%$ & $0.8 \%(p=0.12) *$ & $3.3 \%(p=0.75)^{*}$ & $6.3 \%$ \\
\hline
\end{tabular}

Tabla 2. Porcentajes de casos de parto prematuro en cada grupo, antes de las 37 y 35 semanas de gestación. Prof $=$ profilaxis. Grupo ref. $=$ grupo de referencia. *Valores p calculados respecto al grupo profilaxis + placebo. (Jeffcoat y cols. 2003).

\begin{tabular}{|ccc|}
\hline & $\begin{array}{c}\text { RAR }+ \\
\text { placebo }\end{array}$ & $\begin{array}{c}\text { RAR + } \\
\text { metronidazol }\end{array}$ \\
PP<37 semanas & 0.5 & 1.4 \\
PP<35 semanas & 0.2 & 0.7 \\
\hline
\end{tabular}

Tabla 3. Risk ratios calculadas respecto al grupo profilaxis + placebo (Jeffcoat $y$ cols 2003).

cidos para PP/BPN. El factor de riesgo que tuvo el riesgo relativo más elevado fue la enfermedad periodontal con un valor de 5.99, por encima de otros como tabaquismo, PP/BPN previo, menos de 6 visitas prenatales o baja ganancia de peso por parte de la madre.

Jeffcoat y colaboradores realizaron en 2003 un nuevo estudio controlado randomizado. La muestra contó con un $85 \%$ de mujeres de raza afroamericana y una edad media de 22.5 años. Trescientas sesenta y seis mujeres con periodontitis (aceptando como tal la presencia de más de tres sitios con una pérdida de inserción clínica de al menos $3 \mathrm{~mm}$ ) fueron distribuidas aleatoriamente en tres grupos de tratamiento siguiendo una estratificación por: a) historia previa de parto prematuro espontáneo antes de las 35 semanas de gestación; b) índice de masa corporal menor de 19.8; c) vaginosis bacteriana confirmada mediante tinción de Gram. Los grupos recibieron respectivamente las siguientes terapias: 1) profilaxis (tartrectomía supragingival y pulido con copa de goma) y administración de cápsula placebo tres veces al día; 2) raspado y alisado radicular (RAR) y administración de cápsula placebo tres veces al día; 3) RAR y administración de metronidazol $250 \mathrm{mg}$ tres veces al día durante una semana. Los tres grupos recibieron instrucciones de higiene oral. Otras 723 mujeres con periodontitis que participaban en un estudio observacional sirvieron como grupo de referencia. Las mujeres de este grupo pertenecían a la misma población que las que formaban los grupos de tratamiento. Los tres grupos de tratamiento fueron comparados, no hallándose diferencias estadísticamente significativas en cuanto a diversos factores de riesgo, datos demográficos y estatus periodontal. En la tabla 2 se muestran los porcentajes de pacientes en cada grupo que dieron a luz antes de 37 y 35 semanas de gestación. La única diferencia estadísticamente significativa se encontró entre los dos grupos que recibieron RAR, además de metronidazol y placebo respectivamente $(p=0.02)$, mostran- do el grupo RAR + metronidazol una tasa de partos prematuros superior. En la tabla 3 se muestran las risk ratios respecto al grupo profilaxis + placebo.

\section{DISCUSIÓN}

Los datos epidemiológicos revisados dejan constancia del problema de salud pública que supone el PP/BPN, y por tanto de la importancia de su prevención. Uno de los objetivos del presente artículo es revisar la evidencia científica existente respecto a la asociación entre enfermedad periodontal y parto prematuro. La literatura que se ha consultado en esta revisión no sólo constata el papel de la enfermedad periodontal como factor de riesgo para la ocurrencia del PP/BPN, sino que en determinados casos la señala incluso como uno de los más importantes.

De entre los numerosos datos publicados al respecto cabe destacar que en un estudio controlado randomizado se ha observado que las mujeres embarazadas con enfermedad periodontal tienen un riesgo casi 6 veces mayor de sufrir PP/BPN que las mujeres periodontalmente sanas, siendo el factor de riesgo más fuerte de los estudiados por encima del tabaquismo, baja ganancia de peso materno o menos de seis consultas prenatales (López y cols. 2001). Los hallazgos de otros estudios refuerzan notablemente esta afirmación, alcanzándose un riesgo relativo de hasta 7.9 para enfermedad periodontal y PP/BPN (Offenbacher y cols. 1996).

No obstante, es importante aclarar que no todas las poblaciones de mujeres con enfermedad periodontal sufren la misma predisposición al PP/BPN. En un estudio de casos y controles en mujeres bangladeshas residentes en Londres, se obtuvo un riesgo relativo de 0.8 para EP y PP/BPN (Davenport y cols. 1998). Sin embargo, en un estudio de cohortes sobre 1313 mujeres en Alabama, EE.UU., se calculó un riesgo relativo de 2.8-4.1 (Jeffcoat y cols. 2001). En otro estudio de cohortes sobre 812 mujeres embarazadas se observó que casi un tercio $(32.4 \%$ ) de las 407 mujeres de raza negra que participaron en el estudio sufrieron un parto prematuro (Offenbacher y cols. 2001). Se deduce por tanto que determinadas poblaciones tienen un 
mayor riesgo de sufrir un parto prematuro, y que la enfermedad periodontal actúa como factor de riesgo, pero no con la misma intensidad en todas las poblaciones. Una cuestión de suma importancia sería determinar en qué poblaciones la enfermedad periodontal predispone mas fuertemente a sufrir un parto prematuro/de bajo peso.

Además de los factores de riesgo ya conocidos, la literatura revisada aporta indicios acerca de varias circunstancias que podrían también tener un papel en la etiopatogenia del PP/BPN, algunos de ellos de especial interés desde el punto de vista de la periodoncia. La detección de anticuerpos IgM específicos frente a Campylobacter rectus en muestras de sangre del cordón umbilical apunta a una mayor capacidad de este microorganismo para alcanzar el feto atravesando la barrera placentaria (Offenbacher y cols. 2001). La observación en placa subgingival de madres prematuras de niveles significativamente más elevados de este organismo frente a otros patógenos periodontales (Mitchell-Lewis y cols. 2001) refuerza esta sospecha. La comparación de los niveles de anticuerpos IgM específicos frente a 13 patógenos periodontales en muestras de sangre de cordón umbilical reveló un predominio de IgM específica frente a C. rectus (Offenbacher y cols. 1999). Por otra parte se ha observado que algunas especies no humanas del género Campylobacter son capaces de atravesar la barrera placentaria e inducir el parto prematuro y/o el aborto en animales de ganado (Coid y Fox, 1983). Bacteroides forsytus también ha sido hallado en cantidades significativamente elevadas en madres prematuras (Offenbacher y cols. 1998, Mitchell-Lewis y cols. 2001). Este podría ser un interesante punto de partida para futuros estudios. De confirmarse que estos patógenos suponen un riesgo añadido en la ocurrencia del PP/BPN, sería de gran importancia la toma de muestras microbiológicas periodontales en pacientes embarazadas con enfermedad periodontal como prueba diagnóstica de despistaje.

Otra situación que podría tener importancia en el contexto de la relación entre enfermedad periodontal y $\mathrm{PP} / \mathrm{BPN}$ es el caso de pacientes con una respuesta inflamatoria hipersensible, condición que aumentaría el riesgo de sufrir tanto enfermedad periodontal como PP/BPN (Offenbacher y cols. 1996). Esta circunstancia es a menudo detectada a través del diagnóstico periodontal, y podría ser importante la interconsulta con el ginecólogo de cara a la prevención del PP/BPN.

Otro de los propósitos de la presente revisión era analizar la evidencia científica en lo relativo al papel del tratamiento periodontal como medida de prevención del parto prematuro. Se han encontrado múltiples justificaciones para la aplicación de dicha terapia. Se ha demostrado el papel etiológico que las infecciones remotas como la enfermedad periodontal ejercen en los mecanismos que conducen al parto prematuro, actuando como reservorio de lipopolisacárido y de mediadores inflamatorios capaces de alcanzar la placenta por vía sanguínea (Offenbacher y cols. 1996, Offenbacher y cols. 1998, Offenbacher y cols. 1999, Jeffcoat y cols 2001 a, Gibbs 2001). Se ha demostrado la capacidad de determinados patógenos periodontales o de sus componentes de alcanzar la unidad fetoplacentaria e inducir la síntesis de mediadores inflamatorios que participan en la patogenia del PP/BPN (Offenbacher y cols. 1996, Offenbacher y cols. 1999, Offenbacher y cols. 2001, Jeffcoat y cols 2001 a). La presencia de enfermedad periodontal en fase activa incrementa el riesgo de exposición sistémica al lipopolisacárido y a distintos mediadores inflamatorios (Offenbacher y cols. 1998). Estas observaciones indican la relevancia que cobra el control de la infección e inflamación periodontales en pacientes gestantes, control que es posible por medio del tratamiento periodontal.

En un estudio de cohortes sobre más de 800 mujeres embarazadas se ha observado que un $47.2 \%$ de las madres prematuras experimentó una progresión de su enfermedad periodontal durante el embarazo, hallándose una fuerte significación estadística $(\mathrm{p}=$ 0.0012 ) en la asociación entre estas dos circunstancias (progresión de la EP y parto prematuro/de bajo peso) (Offenbacher y cols. 2001). Estos datos reflejan la importancia de evitar dicha progresión, lo cual también es posible mediante el tratamiento periodontal y una adecuada instrucción en el control de placa.

Se ha sugerido que exposiciones intermitentes a corto plazo a factores de riesgo durante el embarazo pueden jugar un papel importante en el desencadenamiento del parto pretérmino (Offenbacher y cols. 2001). Esta observación recalca la necesidad de un correcto control de placa y un adecuado programa de mantenimiento durante el embarazo, además del tratamiento periodontal en los casos que lo requieran.

Se ha observado también que las madres que no desarrollan una respuesta humoral sistémica frente a determinados patógenos periodontales tienen una tasa de parto prematuro significativamente mayor, lo que sugiere que dicha respuesta de anticuerpos puede tener una función protectora para el feto. Este hallazgo ha llevado a los investigadores a pensar que un tratamiento que refuerce la respuesta de anticuerpos por parte de la madre, como el raspado o alisado radicular o incluso la vacunación, podría reducir los efectos adversos de la enfermedad periodontal sobre el embarazo (Offenbacher 2001).

La evidencia científica más importante a este respecto es aportada por el estudio controlado randomizado 
de López y colaboradores. Sus resultados señalan que las mujeres gestantes con enfermedad periodontal no tratada tienen un riesgo de sufrir PP/BPN 5.49 veces mayor que las embarazadas con enfermedad periodontal tratada antes de las 28 semanas de gestación ( $p$ = 0.001) (López y cols. 2001).

Pero aunque disponemos de evidencia científica respecto a que la enfermedad periodontal no tratada incrementa el riesgo de parto prematuro y parece que los primeros estudios de intervención indican que el tratamiento de la infección periodontal reduce la tasa de PP/BPN, subsiste una cuestión fundamental por resolver: ¿qué manera de tratar la enfermedad periodontal es más eficaz en la prevención del parto prematuro? Serán necesarios nuevos estudios de tratamiento y la comparación entre distintas modalidades terapéuticas para dar respuesta a esta pregunta.

López y colaboradores obtuvieron resultados satisfactorios realizando raspado y alisado radicular, una correcta instrucción en el control de placa y un adecuado programa de mantenimiento durante el embarazo, además de una terapia antibiótica (metronidazol $250 \mathrm{mg}$ y amoxicilina $500 \mathrm{mg}$ tres veces al día durante una semana) en los casos de periodontitis agresiva severa (López y cols. 2001). Sin embargo, Jeffcoat y colaboradores no encontraron diferencias significativas entre la aplicación de profilaxis y la de raspado y alisado radicular con o sin metronidazol.

Por otra parte, se ha investigado abundantemente acerca de la prevención del parto pretérmino a través del tratamiento con antibióticos, pero los resultados de los distintos estudios son discordantes. La explicación de esta falta de concordancia puede hallarse en el hecho de que el parto prematuro tiene una etiología multifactorial, y en los resultados de los estudios los casos no provocados por una infección podrían haber diluido el efecto del tratamiento antibiótico. Otra posibilidad es que los antibióticos empleados en los estudios simplemente no fueran los adecuados (Gibbs 2001). En cualquier caso, el uso de antibióticos en la prevención del parto prematuro ha resultado en ocasiones en serias infecciones causadas por organismos resistentes tanto en la madre como en el hijo (McDuffie y cols. 1993), así como en un aumento significativo de la incidencia de enterocolitis necrotizante neonatal (Owen y cols. 1993). Se ha observado que el metronidazol no es eficaz en la reducción de la tasa de PP en mujeres con vaginosis bacteriana (Carey y cols. 2000), obteniéndose incluso tasas significativamente mayores que las de un grupo control tratado con un placebo (Klebanoff y cols, 2001). Asimismo, se ha observado en el estudio controlado randomizado de Jeffcoat y colaboradores que la administración de metronidazol reduce la eficacia del raspado y alisado radicular en la prevención del parto prematuro
(Jeffcoat y cols. 2003). Así pues, en cuanto a la terapia antibiótica dentro del tratamiento periodontal, se aconseja ser especialmente cauteloso en la administración de estos fármacos a mujeres gestantes hasta que nuevos estudios permitan establecer protocolos seguros y libres de riesgos.

\section{CONCLUSIONES}

La relación entre la enfermedad periodontal y el parto prematuro/de bajo peso ha sido explicada a través de múltiples vías. Los numerosos estudios al respecto aportan evidencia científica suficiente para considerar que existe dicha relación y que la enfermedad periodontal es un factor de riesgo independiente para parto prematuro/de bajo peso. Las diferencias obtenidas por distintos estudios sobre diferentes poblaciones ponen de manifiesto que la enfermedad periodontal supone un mayor riesgo de sufrir un parto pretérmino en determinadas poblaciones. Serán necesarios nuevos estudios para concretar cuáles son esas poblaciones. Asimismo, los hallazgos de distintos estudios permiten sospechar una especial implicación de algunos patógenos periodontales como Campylobacter rectus y Bacteroides forsytus en la patogenia del parto prematuro/de bajo peso, generándose nuevas hipótesis que requerirán también de los correspondientes estudios.

El tratamiento periodontal de las pacientes gestantes con enfermedad periodontal parece tener claros beneficios. Los resultados de diversos estudios desaconsejan la administración de antibióticos en mujeres embarazadas con el fin de prevenir el parto prematuro. No obstante serán necesarios nuevos estudios controlados randomizados para ratificar la justificación y la necesidad del tratamiento periodontal en estas pacientes, así como para determinar qué tipo de terapia periodontal es más eficaz en la prevención del parto prematuro/de bajo peso y establecer un protocolo de actuación ante las pacientes gestantes con enfermedad periodontal.

\section{SUMIMARY}

Despite all efforts made for pregnancy and labour care, the incidence of preterm births has not decreased in the last two decades. Prematurity implies a serious threat to the health of the newborn, representing an important percentage of the perinatal health care costs. Scientific research has revealed multiple mechanisms through which remote infections such as 
periodontal disease can participate in the pathogenesis of preterm birth. Recent studies have demonstrated that periodontal disease is a risk factor for preterm birth, even with higher odds ratios than those calculated for traditional risk factors such as tobacco consumption, low maternal weight or alcohol consumption. It has been suggested that the prevention and treatment of periodontal disease in pregnant women could contribute to the prevention of preterm birth. This paper reviews the scientific evidence in the literature regarding this asociation and the role of periodontal therapy in preventing preterm birth.

\section{KEY WORDS}

Premature birth, low birth weight, periodontal disease, pregnancy, evidence based.

\section{AGRADECIMIENTOS}

Los autores agradecen al Dr. Pluvio Coronado su colaboración.

\section{CORRESPONDENCIA}

Juan Flores Guillén.

C/ Hilarión Eslava $142^{\circ} \mathrm{D}$

28015 Madrid

Tel.: 915493961

E-mail: juancho.flores@telefonica.net

\section{BIBLIOGRAFÍA}

1. Armitage G.C. Periodontal disease and pregnancy: discussion, conclusions and recomendations. Ann Periodontol 2001; 6:189-92.

2. Morales W.J., Schorr S., Albritton J. Effects of metronidazole in patients with preterm birth in preceding pregnancy and bacterial vaginosis: a placebo controlled double blind study. Am J Obstet Gynecol 1994; 171: 345-9.

3. Hillier S.L., Witkin S.S., Krohn M.A., Watts D.H., Kiviat N.B., Eschenbach D.A. The relationship of amniotic fluid cytokines and preterm delivery, amniotic fluid infection, histologic chorioamnionitis and chorioamnion infection. Obstet Gynecol 1993; 81: 941-8.

4. Romero R., Yoon B.H., Mazor M., Gomez R., et al. The diag- nostic and prognostic value of amniotic fluid white blood cell count, glucose, interleukin-6, and Gram stain in patients with preterm labor and intact membranes. Am J Obstet Gynecol 1993; 169: 805-16.

5. Dasanayake A.P. Poor periodontal health of the pregnant woman as a risk factor for low birth weight. Ann Periodontol 1998; 3: 206-12.

6. Jeffcoat M.K., Geurs N.C., Reddy M.S., Goldenberg R.L., Hauth J.C. Current evidence regarding periodontal disease as a risk factor for preterm birth. Ann Periodontol 2001; 6: 183-8. (Jeffcoat 2001a).

7. Jeffcoat M.K., Geurs N.C., Reddy M.S., Cliver S.P., Goldenberg R.L., Hauth J.C. Periodontal infection and preterm birth. Results of a prospective study. J Am Dent Assoc 2001; 132: 875-80. (Jeffcoat 2001b).

8. Davenport E.S., Williams C.E., Sterne J.A., Sivapathasundram V., Fearne J.M., Curtis M.A. The East London Study of maternal chronic periodontal disease and preterm low birth weight infants: study design and prevalence data. Ann Periodontol 1998 Jul; 3 (1): 213-21.

9. Muñiz García J., Gabriel Sánchez R. Tipos de estudios en investigación clínica. Medicine 2003; 8 (118): 6332-6.

10. Mitchell-Lewis D., Engebretson S.P., Chen J., Lamster I.B., Papapanou PN. Periodontal infections and pre-term birth: early findings from a cohort of young minority women in New York. Eur J Oral Sci. 2001; 109 (1): 34-9.

11. Coronado P.J., Asenjo J.E., Fasero M. et al ¿Altera la vía de parto los resultados de los grandes pretérminos? TokoGin Práct 1998; 57: 233-40.

12. Jimeno J.M., Coronado P.J., Cristóbal I, Escudero M. Estudio de la vía de parto como predictor de la muerte neonatal en recién nacidos entre 500 y 1500 gramos. Actualidad Obstétrico-Ginecológica 1997; 9 (4): 168-75.

13. World Health Organization, Expert Committee on Maternal and Child Health. Public health aspect of low birth weight. Geneva, Switzerland; WHO technical report series, no. 27, 1950.

14. Krueger T.M., Scholl T.O. Adequacy of prenatal care and pregnancy outcome. J Am Osteopath Assoc 2000; 100: 485-92.

15. Stevenson D.K., Wright L.L., Lemons J.A., et al. Very low birth weight outcomes of the National Institute of Child Health and Human Development Neonatal Research Network, January 1993 through December 1994. Am J Obstet Gynecol 1998; 179: 1632-9.

16. Coid C.R., Fox H. Short review: Campylobacters as placental pathogens. Placenta 1983; 4: 295-305.

17. McDuffie R.S. Jr, McGregor J.A., Gibbs R.S. Adverse perinatal outcome and resistant Enterobacteriaceae after antibiotic usage for premature rupture of the membranes and group B streptococcus carriage. Obstet Gynecol 1993; 82: 487-9. 
18. Owen J., Groome L.J., Hauth J.C. Randomized trial of prophylactic antibiotic therapy after preterm amnion rupture. Am J Obstet Gynecol 1993; 169: 976-81.

19. Lorenz J.M., Wooliever D.E., Jetton J.R., Pannete N. A quantitative review of mortality and developmental disability in extremely premature newborns. Arch Ped Adolescent Med 1998; 152: 425-35.

20. Carey J.C., Klebanoff M.A., Hauth J.C. et al. Metronidazole to prevent preterm delivery in pregnant women with asymptomatic bacterial vaginosis. N Engl J Med 2000; 24: 3428: 581-3.

21. Klebanoff M.A., Carey J.C., Hauth J.C. et al. Failure of metronidazole to prevent preterm delivery in pregnant women with asymptomatic Trichomonas vaginalis infection. N Engl J Med 2001; 345: 487-93.

22. Collins J.G., Windley H.W. 3rd, Arnold R.R., Offenbacher S. Effects of a Porphyromonas gingivalis infection on inflammatory mediator response and pregnancy outcome in hamsters. Infect Immun. 1994; 62: 4356-61.

23. Collins J.C., Kirtland B.C., Arnold R.R. et al. Experimental periodontitis retards hamster fetal growth. J Dent Res 1995; 1171: 74 .

24. Alexander G.R., Korenbrot C.C. The role of prenatal care in preventing low birth weight. Fut Child 1995; 5: 103120.

25. Minkoff H., Grunebaum A.N., Schwarz R.H. et al. Risk factors for prematurity and premature rupture of membranes: a prospective study of the vaginal flora in pregnancy. Am J Obstet Gynecol 1984 15; 150 (8): 965-72.

26. McDonald HM, O'Loughlin JA, Jolley P et al.Vaginal infection and preterm labour. Br J Obstet Gynaecol 1991; 98:427-435.

27. Stetzer B.P., Mercer B.M. Antibiotics and preterm labor. Clin Obstet Gynecol 2000; 43: 809-17.

28. Socransky S.S., Haffajee A.D., Cugini M.A., Smith C., Kent Jr R.L. Microbial complexes in subgingival plaque. J Clin Periodontol 1998; 25: 134-44.

29. Paquette D.W., Madianos P., Offenbacher S., Beck J.D., Williams RC. The concept of "risk" and the emerging discipline of periodontal medicine. J Contemp Dent Pract 1999 15; 1(1):1-8.
30. Gibbs R.S. The relationship between infections and adverse pregnancy outcomes: an overview. Ann Periodontol 2001; 6 (1): 153-63.

31. Offenbacher S., Lieff S., Boggess K.A. et al. Maternal periodontitis and prematurity. Part I: Obstetric outcome of prematurity and growth restriction. Ann Periodontol 2001; 6 (1): 164-74.

32. Madianos P.N., Lieff S., Murtha A.P. et al. Maternal periodontitis and prematurity. Part II: Maternal infection and fetal exposure. Ann Periodontol 2001; 6 (1): 175-82.

33. Offenbacher S., Katz V., Fertik G. et al. Periodontal infection as a posible risk factor in preterm low birth weight. J Periodontol 1996; 67: 1103-13.

34. Lopez N.J., Smith P.C., Gutierrez J. Periodontal therapy may reduce the risk of preterm low birth weight in women with periodontal disease: a randomized controlled trial. J Periodontol 2002; 73 (8): 911-24.

35. Jeffcoat M.K., Hauth J.C., Geurs N.C. et al. Periodontal disease and preterm birth: results of a pilot intervention study. J Periodontol 2003; 74 (8): 1214-8.

36. Hack M., Taylor G., Klein N.K. et al. School age outcomes in children with birth weights below $750 \mathrm{~g}$. N Engl J Med 1994; 331: 753-9

37. Hack M., Klein N.K., Taylor G. Long-term developmental outcomes of low birth weight infants. Fut Child 1995; 5: 176-96.

38. Woolf S.H., Battista R.N., Anderson G.M., Logan A.G., Wang E. Assesing the clinical effectiveness of preventive maneuvers: analytic principles and systematic methods in reviewing evidence and developing clinical practice recommendations. A report by the Canadian Task Force on the periodic health examination. J Clin Epidemiol 1990; 43 (9):891-905.

39. Sackett D. Rules of evidence and clinical recommendations. Can J Cardiol 1993; 9 (6): 487-9.

40. Lofthus J.E., Waki M.Y., Jolkovsky D.L. et al. Bacteremia following subgingival irrigation and scaling and root planing. J Periodontol. 1991 Oct; 62 (10): 602-7.

41. Waki M.Y., Jolkovsky D.L., Otomo-Corgel J. et al. Effects of subgingival irrigation on bacteremia following scaling and root planing. J Periodontol. 1990 Jul; 61 (7): 405-11. 